\title{
Can We Correlate the Spider's Brain Activity to it Spinning Web Activity?
}

\author{
Hamidreza Namazi \\ 167 Division Ave Brooklyn, New York 11211, USA
}

*Corresponding Author: Hamidreza Namazi, 167 Division Ave Brooklyn, New York 11211, USA, E-mail:M080012@e.ntu.edu.sg

Received: June 17, 2017

Analyzing the brain activity in human and animals is the main research topic in neuroscience [1]. In this way, the analysis of spider's brain has aroused the attention of some scientists. On the other hand, building fractal webs as an important action of spiders (which mainly done for catching their food) has been studied widely. However, no one has discovered any relation between the structure of spider's brain signal (as the feature of brain activity) and the structure of its web. Now, the question, which arises here, is that how spider's brain activity affects its web. Here, in order to make a link between these two features, we benefit from the concept of fractal.

Fractal theory [2] can be used to study the scaling properties of the spider's web. A fractal is a natural phenomenon or a mathematical set that exhibits a repeating pattern that displays at every scale (self-similar). The scaling rules are characterized by "scaling exponents" (dimension). "Simple" regular fractals have integer scaling dimensions. Complex selfsimilar objects have non-integer dimension. Fractals can be defined as geometric objects whose scaling exponent (dimension) satisfies the Szpilrajn inequality [3]:

$\aleph \geq D_{-}$T

Where Nis the scaling exponent (dimension) of the object and $\mathrm{D}_{\mathrm{T}}$ is its topological dimension, that is, Euclidean dimension of units from which the fractal object is built. In fact, Fractal and Euclidean geometries are conjugate approaches. Fractal geometry builds complex objects by applying simple processes to complex building blocks; Euclidean geometry uses simpler building blocks but frequently requires complex building processes [4]. Fractal dimension can be viewed as an index of complexity, which shows how a detail in a pattern changes with the scale at which it is measured. Fractal approach has been used widely in order to study the complexity of different processes [5-9].

Here, for the first time we aim to investigate the relation between spider's brain activity and its web. As spider's brain signal and web is complex, and the activity of spider is organized by its brain, we propose that we can benefit from the concept of complexity and hypothesize that the complexity of spider's brain signal should affect its web complexity. To test this hypothesis, for the first time we can investigate the correlation between the fractal nature of the spider's web and the fractal structure of its brain signal in different conditions.

As a suggestion, based on the research done by Witt [10] that stated the spider's brain activity and the pattern of its web could be changed using psychoactive drugs, we can analyze the activity of spider's brain signal versus its spinned web pattern using fractal dimension, in different levels of brain activity which caused by the applied drug. In this way, we can correlate the spider's brain signal to its web. Although the main purpose of such study is to analyze the relation between the spider's brain activity and its web, but based on the previous work on the analysis of the influence of psychoactive drugs on spiders, where Witt [10] stated that spiders behaviors (such as drowsiness and walking) on psychoactive drugs are like humans on drugs, our investigation on the relation between the spider' brain activity and its web on psychoactive drugs may explain the relation between human brain activity and his behavior in case of psychoactive drug 
application, where doing these experiments on human is restricted by law and also doing the same experiments for higher animals is expensive and time consuming.

\section{REFERENCES}

[1] Namazi H, Kulish VV. A mathematical based calculation of a myelinated segment in axons. ComputBiol Med. 2013 Jul; 43(6):693-8. doi: 10.1016/j.compbiomed.2013.03.005.

[2] Parke, G. A. R. \& Disney, P. Space Structures 5 (Thomas Telford, 2002)

[3] Kulish, V.V. Partial Differential Equations (Pearson, 2010)

[4] Namazi H, Akrami A, Haghighi R, Delaviz A, Kulish V.V. Analysis of the Influence of Element's Entropy on the Bulk Metallic Glass (BMG) Entropy, Complexity and Strength. Metallurgical and Materials Transactions A. 2016; DOI: 10.1007/s11661-016-3870-3.

[5] Anandarup, M., Nidhi, P. \&Anirban, R. Heart murmur detection using fractal analysis of phonocardiograph signals. Int. J. Comput. Appl. 88; DOI: 10.5120/15407-3928 (2014).

[6] Kyung-Hoe, H. et al. Fractal analysis of mandibular trabecular bone: optimal tile sizes for the tile counting method. Imaging Sci. Dent. 41, 71-78 (2011).

[7] Namazi, H. \&Kulish, V. V. Fractional Diffusion Based Modelling and Prediction of Human Brain Response to External Stimuli. Comput. Math. Methods Med.2015, 148534; DOI:10.1155/2015/148534 (2015).

[8] Namazi, H. \&Kiminezhadmalaie, M. Diagnosis of Lung Cancer by Fractal Analysis of Damaged DNA. Comput. Math. Methods Med. 2015; 1-13 (2015).

[9] Seetharaman, K., Namazi, H. \&Kulsih, V. V. Phase lagging model of brain response to external stimuli-modeling of single action potential. Comput. Biol. Med. 42, 857-862 (2012).

[10]Rainer, F. F. Biology of spiders (Oxford University Press, 2010).

Citation: Hamidreza Namazi, Can We Correlate the Spider's Brain Activity to it Spinning Web Activity?. ARC Journal of Neuroscience. 2017; 2(1):17-18. doi:dx.doi.org/10.20431/2456-057X.0201005.

Copyright: () 2017 Authors. This is an open-access article distributed under the terms of the Creative Commons Attribution License, which permits unrestricted use, distribution, and reproduction in any medium, provided the original author and source are credited. 